\title{
Postprandial activation of metabolic and inflammatory signalling pathways in human peripheral mononuclear cells
}

\author{
Kerstin Ehlers $^{1,2,3}$, Tina Brand ${ }^{2,3,4}$, Adina Bangert ${ }^{2,5}$, Hans Hauner ${ }^{1,2,3,4,6} \dagger$ and Helmut Laumen ${ }^{1,2,3,6,7 *} \dagger$ \\ ${ }^{1}$ Else Kröner-Fresenius-Centre for Nutritional Medicine, Chair of Nutritional Medicine, Technische Universität München \\ (TUM), Gregor-Mendel-Strasse 2, 85350 Freising-Weihenstephan, Germany \\ ${ }^{2}$ ZIEL - Research Centre for Nutrition and Food Sciences, Technische Universität München (TUM), \\ Freising-Weibenstephan, Germany \\ ${ }^{3}$ Clinical Cooperation Group Nutrigenomics and Type 2 Diabetes, Technische Universität München (TUM) \\ and Helmboltz Zentrum München (HMGU), Freising-Weihenstephan and Neuberberg, Germany \\ ${ }^{4}$ Else Kröner-Fresenius-Centre for Nutritional Medicine, Klinikum rechts der Isar, \\ Technische Universität München (TUM), München, Germany \\ ${ }^{5}$ Molecular Nutrition Unit, Technische Universität München (TUM), Gregor-Mendel-Strasse 2, \\ 85350 Freising-Weibenstephan, Germany \\ ${ }^{6}$ German Centre for Diabetes Research (DZD), Neuberberg, Germany \\ ${ }^{7}$ Institute of Experimental Genetics, Helmboltz Zentrum München (HMGU), Neuberberg, Germany
}

(Submitted 7 March 2013 - Final revision received 23 December 2013 - Accepted 2 January 2014 - First published online 28 February 2014)

\section{Abstract}

High-fat, high-carbohydrate (HFHC) meals induce an inflammatory response in mononuclear cells (MNC). Here, we studied the interaction between metabolic and inflammatory signalling pathways by the measurement of postprandial effects of three different test meals on intracellular Akt, S6 kinase (S6K)/mammalian target of rapamycin and NF-кB signalling in human MNC. We recruited six healthy, lean individuals. Each individual ingested three different meals in the morning separated by at least $3 \mathrm{~d}$ : a HFHC meal; an oral lipid-tolerance test meal; a healthy breakfast. Blood samples were obtained before and 1, 2, 4, 6 and $8 \mathrm{~h}$ after ingestion. Plasma insulin and IL- 6 levels were measured. Intracellular metabolic and inflammatory signalling pathways were assessed by measuring the phosphorylation of Akt kinase and $\mathrm{S} 6 \mathrm{~K}$, the degradation of inhibitory $\kappa \mathrm{B}-\alpha(\mathrm{I} \kappa \mathrm{B}-\alpha)$ protein and the DNA binding activity of NF- $\kappa \mathrm{B}$ in MNC. mRNA expression levels of the Akt and NF- $\mathrm{BB}$ target genes Mn superoxide dismutase (MnSOD), CC-chemokine-receptor 5 (CCR5), intercellular adhesion molecule 1 (ICAM-1) and plasminogen activator inhibitor-1 (PAI-1) were measured by quantitative RT-PCR. We found a positive correlation

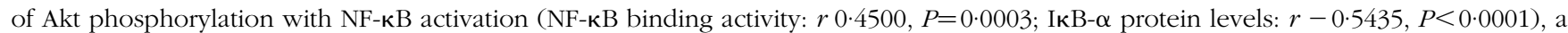
negative correlation of plasma insulin levels with NF- $\mathrm{BB}$ binding activity $(r-0 \cdot 3993, P=0 \cdot 0016)$ and a positive correlation of plasma insulin levels with S6K activation $(r$ 0.4786, $P<0.0001)$. The activation of Akt and pro-inflammatory NF- $\mathrm{B}$ signalling was supported by the up-regulation of the respective target genes $M n S O D$ and CCR5. In conclusion, the present data suggest a postprandial interaction between the metabolic and inflammatory signalling pathways Akt and NF- $\mathrm{BB}$ in MNC.

Key words: Mononuclear cells: Postprandial signalling: Akt: NF-кB: S6 kinase

Numerous lines of evidence indicate that intake of macronutrients activates mononuclear cells $(\mathrm{MNC})^{(1,2)}$ mediated by the $\mathrm{NF}-\kappa \mathrm{B}$ pathway ${ }^{(3)}$. Increasing plasma concentrations of endotoxin (lipopolysaccharide), lipopolysaccharide-binding protein and the expression of its receptor Toll-like receptor 4 induced by high-fat, high-carbohydrate (HFHC) meals contribute to increased oxidative and inflammatory stress ${ }^{(4)}$.
However, the role of other signalling pathways in the postprandial activation of $\mathrm{MNC}$ remains largely elusive. In this context, insulin signalling is a prominent contender, in particular because numerous reports have shown an interrelationship between inflammatory and insulin signalling pathways ${ }^{(5-12)}$.

The Akt and mammalian target of rapamycin (mTOR) pathways, two downstream targets of insulin signal transduction,

\footnotetext{
Abbreviations: FOXO, Forkhead box O; HB, healthy breakfast; HFHC, high-fat, high-carbohydrate; IкB- $\alpha$, inhibitory кB- $\alpha$; mTOR, mammalian target of rapamycin; MnSOD, Mn superoxide dismutase; MNC, mononuclear cells; OLTT, oral lipid-tolerance test; p-S6K, phosphorylated S6 kinase; p-Akt, phosphorylated Akt.
}

*Corresponding author: Dr H. Laumen, fax +49 816171 2097, email helmut.laumen@tum.de

†Both authors contributed equally to this work. 
have been implicated in immune cell homeostasis ${ }^{(13,14)}$; for example, mTOR integrates growth factor signals relayed by insulin and insulin-like growth factors, nutrient signals generated by amino acids and energy signals acting through AMP-activated kinase in $\mathrm{MNC}^{(15)}$. Apart from their metabolic function, the importance of Akt and mTOR signalling in innate and adaptive immunity has been established during recent years. The regulation of both pathways by several intra- and extracellular stimuli including specific receptors, heat shock, oxidative stress and cytokines shows their involvement in a multitude of immunomodulatory processes ${ }^{(13,16-19)}$. Thus, both metabolic signalling pathways provide an important link between metabolism and immune cell function. However, to date, activation of these pathways by defined meals has not been demonstrated, and, moreover, the interaction between metabolic and inflammatory signalling pathways in MNC on intake of macronutrients remains elusive.

The present study was designed to investigate the effect of three different test meals on the interaction between metabolic and inflammatory pathways in MNC. To assess the effects of the test meals, we aimed to further unravel the link between inflammatory and metabolic signalling in MNC. For this purpose, we measured the binding activity of NF- $\kappa \mathrm{B}$, the degradation of NF- $\kappa$ B inhibitory $\kappa \mathrm{B}-\alpha(\mathrm{I} \kappa \mathrm{B}-\alpha)$ protein, the phosphorylation of Akt and S6K as a marker of mTOR activity ${ }^{(20)}$ and the gene expression of both NF-кB and Akt-Forkhead box O (FOXO) target genes. In addition, plasma levels of insulin as a major regulator of Akt and S6K phosphorylation as well as IL-6, a circulating inflammatory cytokine, were determined. In the present study, particular interest was shown to assess the dynamics of pathway activation as well as potential inter-individual differences.

\section{Subjects and methods}

Subjects

A total of six healthy, non-smoking, normal-weight (BMI 24.8 $(\mathrm{SD} 2 \cdot 5) \mathrm{kg} / \mathrm{m}^{2}$ ) males aged $40-53(44.3(\mathrm{SD} 5 \cdot 2)$ ) years were included in the study (for clinical characteristics see online supplementary Table S1). Each subject was subjected to three defined meal tests at the human study centre of the Else Kröner-Fresenius-Centre for Nutritional Medicine (EKFZ) of the Technische Universität München between 08.00 and 09.00 hours after a $12 \mathrm{~h}$ overnight fast on three different days separated by at least $3 \mathrm{~d}$. On each day, fasting blood samples were obtained from the subjects, and then they consumed one of the following meals in the subsequent order: (1) a HFHC meal containing $4630 \mathrm{~kJ}$ of energy ( $48 \%$ as carbohydrates, $11 \%$ as protein and $39 \%$ as fat); (2) a healthy breakfast (HB) containing $2710 \mathrm{~kJ}$ of energy (55\% as carbohydrates, $19 \%$ as protein and $23 \%$ as fat); (3) a standard oral lipid-tolerance test (OLTT) meal consisting of three parts Fresubin Energy Drink (Fresenius Kabi) and one part Calogen (Nutricia) containing on average $3988 \mathrm{~kJ}$ of energy ( $26 \%$ as carbohydrate, $8 \%$ as protein and $70 \%$ as fat). The volume of the liquid meal was calculated for each subject to provide $35 \mathrm{~g}$ fat $/ \mathrm{m}^{2}$ body surface area (for nutrient composition of the meals, see online supplementary Table S2). Blood samples were obtained 1, 2,
3, 4 and $6 \mathrm{~h}$ after ingestion of the $\mathrm{HB}$ and, assuming a prolonged response to high-fat diets, additionally at $8 \mathrm{~h}$ after ingestion of the OLTT meal and the HFHC meal. The study was conducted according to the guidelines laid down in the Declaration of Helsinki, and all procedures involving human subjects were approved by the ethics committee of the Technische Universität München. The study was registered at the German Clinical Trials Register as DRKS00004335 (www.germanctr.de). Written informed consent was obtained from all subjects.

\section{Blood sampling and mononuclear cell isolation}

Venous blood samples were taken from the forearm of the subjects via a vein catheter (18 G $1 \frac{3}{4}$ Vasofix Braunüle) and collected into $4.9 \mathrm{ml}$ EDTA K2-Gel tubes (Sarstedt) as well as $8 \mathrm{ml}$ Vacutainer ${ }^{\circledR} \mathrm{CPT}^{\mathrm{TM}}$ (Cell Preparation Tube; BD) containing sodium heparin as the anti-coagulant. Plasma was obtained by immediate centrifugation (Centrifuge $5702 \mathrm{R}$; Eppendorf AF) at $3000 \mathrm{~g}$ for $10 \mathrm{~min}$ at room temperature, immediately frozen on dry ice and subsequently stored at $-80^{\circ} \mathrm{C}$. The VACUTAINER ${ }^{\circledR}$ Cell Preparation Tubes were processed according to the manufacturer's instructions by centrifugation at $1750 \mathrm{~g}$ for $30 \mathrm{~min}$ at room temperature after which MNC were collected and washed twice with PBS. MNC pellets were frozen in liquid $\mathrm{N}_{2}$ and stored at $-80^{\circ} \mathrm{C}$ until preparation of total protein for the measurement of protein phosphorylation and preparation of RNA for quantitative RT-PCR analysis. Nuclear protein was prepared immediately after the isolation of MNC.

\section{Electrophoretic mobility shift assay of $\mathrm{NF}-\kappa B$}

For the electrophoretic mobility shift assay, nuclear proteins were harvested from MNC. All procedures were performed at $4^{\circ} \mathrm{C}$. Isolated $\mathrm{MNC}$ were suspended in $400 \mu \mathrm{l}$ buffer $\mathrm{A}$ (10 mm-HEPES-KOH, pH $\quad 8, \quad 10 \mathrm{mm-KCl}, \quad 0 \cdot 1 \mathrm{mm-EDTA}$, $0 \cdot 1 \mathrm{~mm}$-ethylene glycol tetraacetic acid, $1 \mathrm{~mm}$-dithiothreitol and $0.5 \mathrm{~mm}$-phenylmethylsulfonyl fluoride). After incubation for $15 \mathrm{~min}$, the cells were lysed by the addition of $15 \mu \mathrm{l}$ of $10 \%$ Nonidet NP-40, and nuclei were isolated by centrifugation at $14000 \mathrm{~g}$ for $3 \mathrm{~min}$. The supernatant was discarded, and the nuclei were resuspended in $50 \mu \mathrm{l}$ buffer C (20 mM-HEPES$\mathrm{KOH}, \mathrm{pH} 8,20 \%$ glycerol, $400 \mathrm{~mm}-\mathrm{NaCl}, 1.5 \mathrm{~mm}-\mathrm{MgCl}_{2}$, $0.2 \mathrm{~mm}$-EDTA, $0.2 \mathrm{~mm}$-ethylene glycol tetraacetic acid, $1 \mathrm{~mm}$-dithiothreitol and $1 \mathrm{~mm}$-phenylmethylsulfonyl fluoride) and lysed by freezing in liquid $\mathrm{N}_{2}$ and subsequent shaking for $15 \mathrm{~min}$. The nuclear protein fraction was collected as the supernatant after centrifugation at $21000 \mathrm{~g}$ for $5 \mathrm{~min}$, frozen in liquid $\mathrm{N}_{2}$ and stored at $-80^{\circ} \mathrm{C}$. Protein concentrations were determined according to the method of Bradford using the Roti $^{\circledR}$ Quant Kit (Carl Roth $\mathrm{GmbH}$ ). ${ }^{32} \mathrm{P}$ end-labelling of a NF- $\kappa \mathrm{B}$ consensus oligonucleotide ( $5^{\prime}$-AGTTGAGGGGACTTTCCCAGGC- $3^{\prime}$ ) using $\left[\gamma^{32}\right.$ P]ATP (Hartmann Analytic) and T4 polynucleotide kinase (Promega) was performed with a commercial kit according to the manufacturer's instructions. Binding of NF- $\mathrm{BB}$ from $2 \mu \mathrm{g}$ of the nuclear extract to $1 \mathrm{ng}$ of labelled oligonucleotides was performed in $10 \mathrm{~mm}$-HEPES ( $\mathrm{pH} 7 \cdot 9$ ), 0.5 mm-EDTA, $25 \mathrm{~mm}$-KCl, $0.5 \mathrm{~mm}$-dithiothreitol, $2 \%$ Ficoll 400, $0.25 \mathrm{mg} \mathrm{BSA} / \mathrm{ml}$ and $50 \mu \mathrm{g} \operatorname{poly}(\mathrm{dI} / \mathrm{dC}) / \mathrm{ml}$ in a total volume 
of $12 \mu \mathrm{l}$ at room temperature for $20 \mathrm{~min}$. Protein-DNA complexes were separated from the free DNA probe by electrophoresis on $6 \%$ native polyacrylamide gels at $180 \mathrm{~V}$ for $2.5 \mathrm{~h}$ at room temperature. The gels were dried, exposed overnight to phosphor screen and subsequently read with a Typhoon 9400 imager (GE Healthcare). The intensity of the protein-DNA complexes was quantified using ImageJ Software (http:// rsbweb.nih.gov/ij/).

\section{Measurement of protein phosphorylation}

For the determination of protein concentration, $1 \times 10^{6} \mathrm{MNC}$ were lysed in $30 \mu l 1 \times$ Milliplex MAP lysis buffer containing freshly prepared protease inhibitors, according to the manufacturer's instructions. Protein concentration was determined using the Pierce BCA Protein Assay Kit (Thermo Fisher Scientific, Inc.). Protein phosphorylation of Akt and S6K was measured by Luminex xMAP technology (Millipore Corporation) in $20 \mu \mathrm{g}$ protein, according to the manufacturer's instructions, using the Bio-Plex 100 System (Bio-Rad Laboratories).

\section{Quantitative RT-PCR}

Total RNA from MNC was isolated using the NucleoSpin Kit (Macherey-Nagel), according to the manufacturer's instructions, and 100 ng RNA was reverse transcribed into complementary DNA (High-Capacity Complementary DNA Reverse Transcription Kit; Applied Biosystems). PCR amplification of human transcripts at baseline and $4 \mathrm{~h}$ after ingestion of the test meal was performed using quantitative PCR (Maxima SYBR-Green, Fermentas; Thermo Fisher Scientific, Inc.) in duplicate using the LightCycler 482 (Roche) with an initial activation of $10 \mathrm{~min}$ at $95^{\circ} \mathrm{C}$ followed by forty cycles of $15 \mathrm{~s}$ at $95^{\circ} \mathrm{C}$ and $40 \mathrm{~s}$ at $61^{\circ} \mathrm{C}$. The results were corrected for phosphoglycerate kinase 1 expression as an internal control $^{(21)}$. The following primers (MWG Biotech) were designed using NCBI primer blast software (http://www.ncbi.nlm.nih.gov/ tools/primer-blast/): CCR5, 5'-CTGAACTTCTCCCCGACAAA-3' (forward) and 5'-TCTCTTCTGGGCTCCCTACA- $3^{\prime}$ (reverse); intercellular adhesion molecule 1, 5'-GGTAAGGTTCTTGCCCACTG-3' (forward) and 5'-TAGAGACCCCGTTGCCTAAA-3' (reverse); Mn superoxide dismutase $(M n S O D), 5^{\prime}$-TCTGTTGGTGTCCAAGGCTC- $3^{\prime}$ (forward) and 5'-TAGTAAGCGTGCTCCCACAC-3' (reverse); phosphoglycerate kinase 1, 5'-CAAGAAGTATGCTGAGGCTGTCA- $3^{\prime}$ (forward) and 5'-CAAATACCCCCACAGGACCAT-3' (reverse).

\section{Measurement of plasma insulin and IL-6 levels}

Plasma insulin and IL-6 levels were measured using commercially available insulin ELISA (Dako) and IL-6 high-sensitivity ELISA (eBioscience) kits, according to the manufacturer's instructions. Intra- and inter-assay CV were as follows: insulin, 7.5 and $9.3 \%$; IL-6, 4.6 and $6.0 \%$, respectively.

\section{Statistical analysis}

All data are expressed as means with their standard errors. Phosphorylated S6K (p-S6K) and Akt (p-Akt) protein levels were normalised to the levels of total protein. All data on $\mathrm{NF}-\kappa \mathrm{B}, \mathrm{I} \kappa \mathrm{B}-\alpha, \mathrm{p}-\mathrm{S} 6 \mathrm{~K}$ and $\mathrm{p}$-Akt levels were normalised to 1 for baseline, and values are expressed as a fold change of the basal level. Statistical analysis was performed with oneway repeated-measures ANOVA for each test meal separately. Additionally, one-sample $t$ tests were used to compare each value with baseline for each test meal separately. Correlation coefficients were calculated using ANCOVA, thus accounting for repeated observations within subjects and meals ${ }^{(22)}$ to evaluate the possible association between measured factors. Plasma insulin level was analysed using two-way repeatedmeasures ANOVA to compare the effect of the three meals and one-way repeated-measures ANOVA with Bonferroni post-test to compare the effects of single meals. Differences in gene expression levels at $4 \mathrm{~h}$ after ingestion of the test meals compared with baseline were assessed by the Wilcoxon matched-pairs test, and differences $4 \mathrm{~h}$ after ingestion were compared using Kruskal-Wallis ANOVA. To compare plasma IL-6 levels at baseline and $6 \mathrm{~h}$ after ingestion of the test meals, two-tailed paired $t$ tests were used for the HFHC meal and the OLTT meal, and Wilcoxon matched-pairs test for the HB. Statistical significance was set as $P<0 \cdot 05$. Statistical analysis was performed using GraphPad Prism 5 (GraphPad Software) and SPSS 21 (SPSS, Inc.). Post hoc calculation of required sample size was calculated using G*Power 3 (http://www. psycho.uni-duesseldorf.de/abteilungen/aap/gpower3/downloadand-register) $^{(23,24)}$.

\section{Results}

Time-dependent postprandial activation of inflammatory signalling pathways in mononuclear cells

To assess pro-inflammatory activation in MNC after ingestion of the test meals, we measured NF-кB DNA-binding activity and ІкB- $\alpha$ protein degradation. Supershift assays revealed two bands containing the NF-кB subunits p50 and p65, respectively (see online supplementary Fig. S1). We observed increased binding activity of NF- $\mathrm{KB}$ following ingestion of the OLTT meal and HFHC meal, and interestingly to a similar extent after ingestion of the HB (Fig. 1(a)). Baseline activity, maximal activation and, in particular, the time point of activation showed a high inter-individual variation after consumption of the three test meals (Fig. 1(a)-(d)). NF- $\kappa$ B binding activity was significantly increased solely in response to the ingestion of the OLTT meal after $4 \mathrm{~h}$, and to the ingestion of the HB after 1 and $4 \mathrm{~h}(P=0.04, P=0.022$ and $P=0.0078$, respectively; Fig. 1(a)). Corresponding to the increase in NF$\kappa \mathrm{B}$ binding activity, we observed a decrease in I $\mathrm{\kappa} \mathrm{B}-\alpha$ total protein levels in response to the ingestion of the OLTT meal, HFHC meal and HB (Fig. 1(e)), but it did not reach overall statistical significance for each meal. A significant difference in mean IкB- $\alpha$ levels was observed in response to the ingestion of the OLTT meal after 2 and $4 \mathrm{~h} \quad(P=0.0220$ and $P=0 \cdot 0181$, respectively), the HFHC meal after 4,6 and $8 \mathrm{~h}$ ( $P=0.0014, P=0.0037, P=0.0203$, respectively) and the $\mathrm{HB}$ after $1 \mathrm{~h}(P=0.016)$ (Fig. 1(e)). Furthermore, we found a highly significant negative correlation $(r-0.3289, P=0 \cdot 0117)$ 


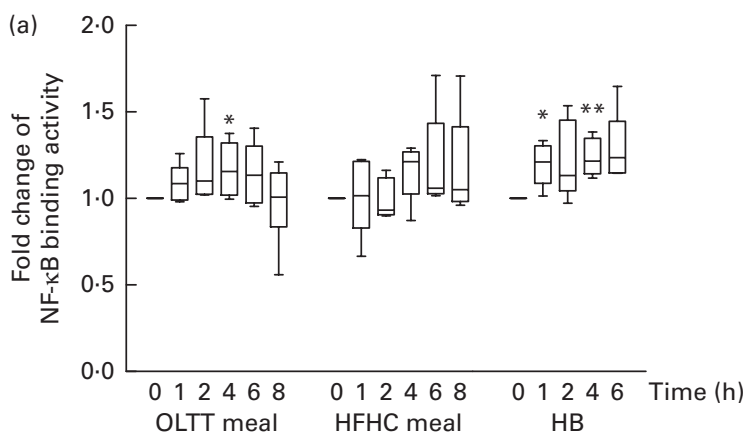

(b)
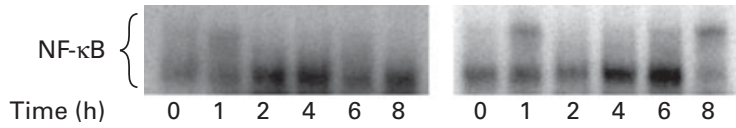

(c)
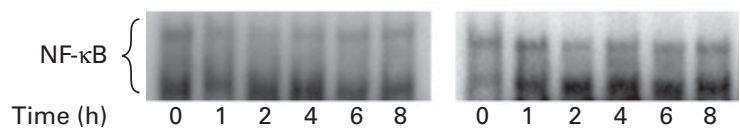

(d)
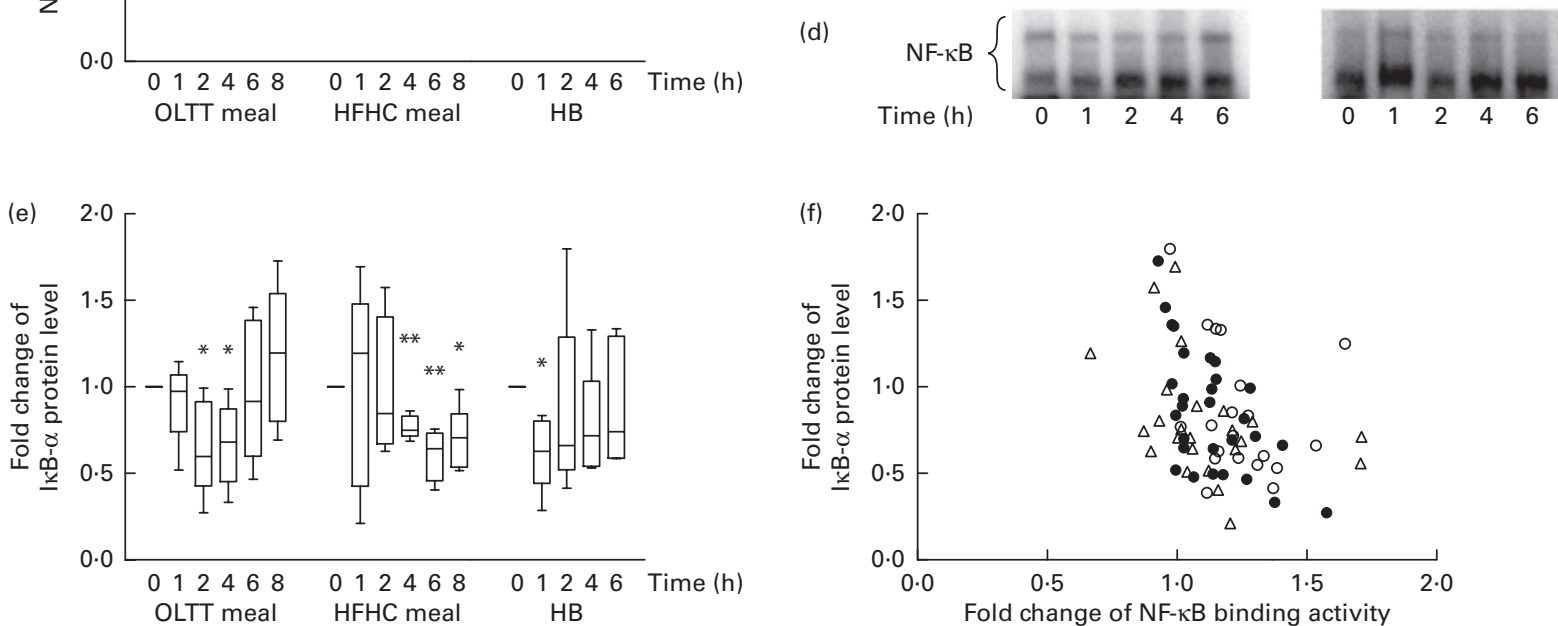

Fig. 1. Postprandial activation of inflammatory signalling pathways in mononuclear cells (MNC). (a) Densitometric analysis of postprandial NF- $\mathrm{kB}$ binding activity in MNC. Two representative electrophoretic mobility shift assay gels showing NF- $\mathrm{kB}$ binding activity levels in MNC nuclear protein extracts following the consumption of (b) an oral lipid-tolerance test (OLTT, $n 6$ ) meal, (c) a high-fat, high-carbohydrate (HFHC, $n 5$ ) meal and (d) a healthy breakfast (HB, $n 5$ ), respectively.

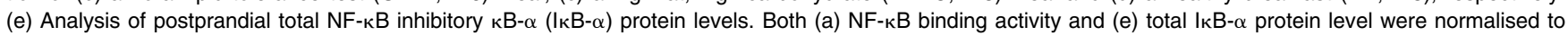
1 for the baseline time point, and values are expressed as a fold change of the basal level (boxes extend from the first quartile to the third quartile; median is indicated as a horizontal line; whiskers are drawn from minimum to maximum values). Value was significantly different from that at baseline: ${ }^{*} P<0.05$, ${ }^{* *} P<0.01$ (one-sample $t$ test). (f) Fold changes of NF-кB binding activity and IкB- $\alpha$ total protein level are plotted against each other for each time point and each subject. Within-subject correlation indicates a negative relationship $(r-0.3289, P=0.0117)$. O, HB; $\bullet$, OLTT; $\triangle$, HFHC meal.

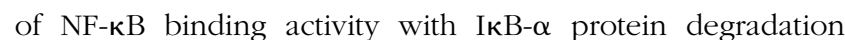
(Fig. 1(f)). NF- $\kappa B$ activation inferred from the correlation of $\mathrm{NF}-\kappa \mathrm{B}$ with I $\mathrm{B}-\alpha$ was significant for the OLTT meal when compared with the HFHC meal and the HB $(P=0.0290$, $P=0.0640$ and $P=0.7281$, respectively; see online supplementary Table S3). Confirming the postprandial activation of inflammatory signalling pathways at a systemic level for all the three test meals, we found a significant increase in plasma IL-6 levels $6 \mathrm{~h}$ after ingestion of the HFHC meal $(P=0.0164)$ and $\mathrm{HB}(P=0.0313)$ and a tendency towards an increase after ingestion of the OLTT meal $(P=0.0547)$ (see online supplementary Fig. S2). There was no significant difference in IL- 6 levels at baseline and $6 \mathrm{~h}$ for all the three test meals studied.

\section{Time-dependent postprandial activation of the metabolic} Akt and 56 kinase signalling pathways in mononuclear cells

Phosphorylation levels of both Akt and S6K increased in total protein extracts from MNC following the ingestion of the OLTT meal, HFHC meal and HB (Fig. 2). We observed a high inter-individual variation in the levels of $56 \mathrm{~K}$ and Akt phosphorylation and thus did not find an overall statistical significance for all the test meals studied. We found a rapid, non-significant increase in $56 \mathrm{~K}$ phosphorylation levels $1 \mathrm{~h}$ after meal ingestion compared with baseline for all the three test meals with a subsequent decrease thereafter (Fig. 2(a)). A significant increase in $\mathrm{p}-\mathrm{S} 6 \mathrm{~K}$ protein levels in response to the ingestion of the OLTT meal was found after $2 \mathrm{~h}$ compared with baseline ( $P=0.05$; Fig. 2(a)). Moreover, we found a rapid increase in Akt phosphorylation levels after ingestion of the OLTT meal and HB in contrast to that of the HFHC meal. Akt phosphorylation appeared to be delayed in response to the ingestion of the HFHC meal (Fig. 2(b)).

\section{Correlation of postprandial metabolic and inflammatory pathway activation}

We calculated the correlations for all measured metabolic and inflammatory signalling pathways (Table 1), and found a significant positive correlation of NF- $\mathrm{BB}$ binding activity with Akt phosphorylation ( $r$ 0.4500, $P=0.0003$; Fig. 3; Table 1). Notably, this association was reflected by a strongly significant negative correlation between Akt phosphorylation and IкB- $\alpha$ protein levels $(r-0.5435, P<0.0001$; Table 1$)$. It should be noted that the correlation of Akt phosphorylation with both measures of NF- $\mathrm{KB}$ activation was significant for most of the test meals (see online supplementary Table S3). Next, we investigated the relationship between the postprandial increase in plasma insulin levels and the measured intracellular parameters of metabolic and inflammatory activation. 

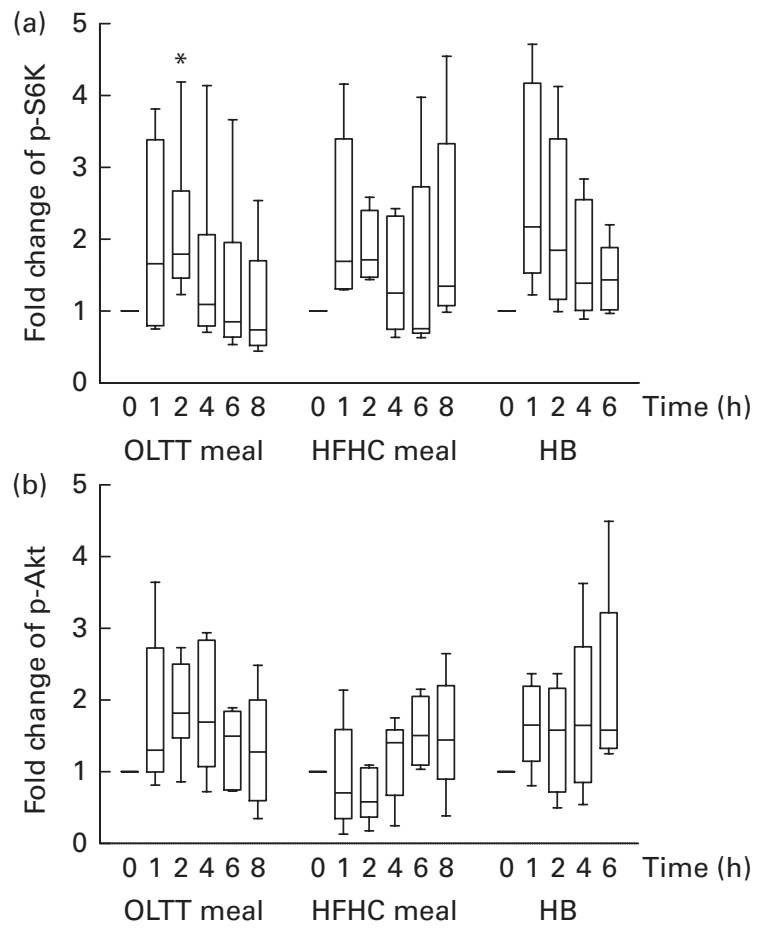

Fig. 2. Postprandial activation of metabolic signalling pathways in mononuclear cells. Changes in the phosphorylation of (a) S6 kinase (p-S6K) and (b) Akt (p-Akt) in response to the consumption of an oral lipid-tolerance test (OLTT, $n$ 6) meal, a high-fat, high-carbohydrate (HFHC, $n$ 5) meal and a healthy breakfast $(\mathrm{HB}, n 5)$ quantified as a ratio of phosphorylated:total protein levels. All values were normalised to 1 for the baseline time point, and values are expressed as a fold change of the basal level. Results are presented as box and whisker plots (boxes extend from the first quartile to the third quartile; median is indicated as a horizontal line; whiskers are drawn from minimum to maximum values). *Value was significantly different from that at baseline $(P \leq 0.05$; one-sample $t$ test).

We found a significant time-dependent increase in plasma insulin levels after ingestion of all the test meals, which was not dependent on the meal type $(P<0.0001, P=0.5920$, respectively; Fig. 4(a)). A direct comparison of the HB-, OLTT meal- and HFHC meal-induced insulin responses at specific time points with baseline levels revealed a significant increase from baseline to $1 \mathrm{~h}$ for all the meals tested $(P<0.0001$ for the HB and HFHC meal and $P<0.001$ for the OLTT meal), to $2 \mathrm{~h}$ for the OLTT meal and HFHC meal ( $P<0.05$ and $P<0.01$, respectively), but not for the $\mathrm{HB}$
(Fig. 4(a)). We found a significant positive relationship of plasma insulin levels with S6K phosphorylation ( $r$ 0.4786, $P<0.0001$; Fig. 4(b); Table 1 ), and a significant negative correlation of plasma insulin levels with NF- $\mathrm{KB}$ binding activity ( $r-0.3993, P=0.0016$; Table 2). The correlation between S6K phosphorylation and plasma insulin levels was significant for the OLTT meal and HB, but not for the HFHC meal, while the correlation between $\mathrm{NF}-\kappa \mathrm{B}$ binding activity and plasma insulin levels was significant for the HB and HFHC meal, but not for the OLTT meal (see online supplementary Table S3). However, no significant correlation was observed for p-Akt, IкB- $\alpha$ and plasma insulin levels (Table 2).

\section{Postprandial changes in gene expression}

To assess the effects of diet-induced inflammatory (NF-кB) and metabolic (Akt) activation on gene expression, mRNA levels for known target genes of NF- $\mathrm{B}$ and FOXO (a transcription factor inhibited by Akt kinase) were studied. The mRNA expression of the FOXO target gene MnSOD (Fig. 5(a)) and that of the NF-кB target gene plasminogen activator inhibitor-1 (PAI-1) (Fig. 5(d)) were significantly downregulated (0.84- and 0.67-fold, respectively) $4 \mathrm{~h}$ after ingestion of the OLTT meal compared with baseline (both $P<0.05$ ). There was a significant down-regulation of MnSOD mRNA expression $(P<0.05)$ by 0.78 -fold $4 \mathrm{~h}$ after ingestion of the HFHC meal compared with baseline (Fig. 5(a)). The mRNA levels of CCR $54 \mathrm{~h}$ after ingestion of the OLTT meal and HB differed significantly $(P<0.05$; Fig. 5(b)). While CCR 5 mRNA levels were increased by $1 \cdot 7$-fold $4 \mathrm{~h}$ after ingestion of the OLTT meal, there was a 0.59 -fold decrease in the mRNA levels of CCR5 after ingestion of the HB (Fig. 5(b)).

\section{Discussion}

The results of the present study show the well-established postprandial activation of inflammatory signalling pathways in MNC at the level of NF- $\mathrm{BB}$ binding activity and IкB- $\alpha$ protein degradation. Moreover, we analysed the interaction between postprandial NF- $\mathrm{KB}$ activation and other metabolic signalling pathways in a defined time course of up to $8 \mathrm{~h}$. We found a correlation of NF- $\mathrm{BB}$ signalling with Akt phosphorylation levels, but not with mTOR/pS6K activation.

Table 1. Within-subject correlations of postprandial activation of metabolic and inflammatory signalling pathways in mononuclear cells*

\begin{tabular}{|c|c|c|c|c|c|c|}
\hline \multirow[b]{2}{*}{ Variables } & \multicolumn{2}{|c|}{ ІкB- $\alpha$} & \multicolumn{2}{|c|}{$\begin{array}{l}\text { NF-кB binding } \\
\text { activity }\end{array}$} & \multicolumn{2}{|c|}{ p-Akt } \\
\hline & $r \dagger$ & $P$ & $r \dagger$ & $P$ & r† & $P$ \\
\hline $\mathrm{p}-\mathrm{S} 6 \mathrm{~K}$ & 0.1339 & 0.3164 & -0.0434 & 0.7462 & -0.0213 & 0.8741 \\
\hline p-Akt & -0.5435 & $<0.0001$ & 0.4500 & 0.0003 & & \\
\hline NF-кB binding activity & -0.3289 & 0.0117 & & & & \\
\hline
\end{tabular}

ІкB- $\alpha$, inhibitory кB- $\alpha$; p-Akt, phosphorylated Akt; p-S6K, phosphorylated S6 kinase.

* Correlation matrix (ANCOVA): correlation coefficients within subjects of the fold change of each measured marker (oral lipid-tolerance test meal, high-fat, high-carbohydrate meal and healthy breakfast) at each time point and each subject were calculated.

† ANCOVA. 


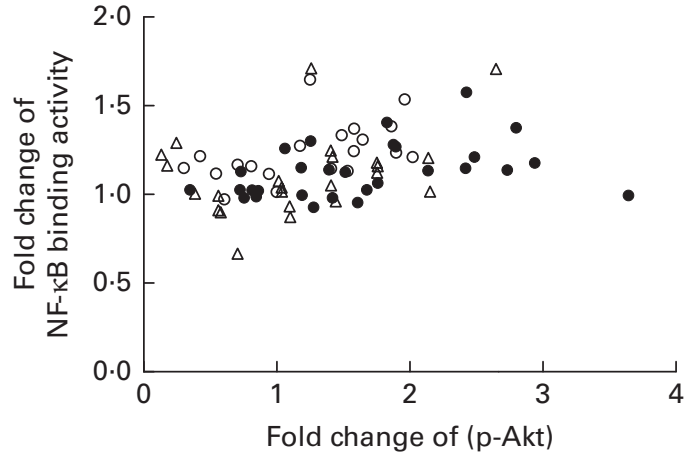

Fig. 3. Within-subject correlation of postprandial activation of inflammatory and metabolic signalling pathways in mononuclear cells. Fold changes of NF-kB binding activity and Akt phosphorylation ( $p-A k t)$ for each measured marker (oral lipid-tolerance test meal, e; high-fat, high-carbohydrate meal, $\triangle$; healthy breakfast, $O$ ) at each time point and each subject are plotted against each other. Within-subject correlation indicates a positive relationship $(r 0.4500, P=0.0003)$.

Moreover, we observed a correlation of plasma insulin levels with both mTOR/S6K phosphorylation levels and NF-кB binding activity, but not with Akt activation or $\mathrm{I} \kappa \mathrm{B}-\alpha$ protein degradation.

We describe herein for the first time a postprandial increase of Akt and S6K phosphorylation levels in MNC by three different test meals. It was shown previously that insulin signalling is impaired at the level of, for example, Akt and insulin receptor phosphorylation levels in the MNC of insulin-resistant subjects $^{(5,25,26)}$. As we excluded a state of insulin resistance by selecting participants with normal body weight, normal glucose metabolism and without a family history of diabetes, future research is needed to assess the role of postprandial Akt activation in the MNC of subjects with impaired insulin sensitivity. Similar to postprandial Akt activation, in the present study, the biological function of postprandial $56 \mathrm{~K}$ activation observed in MNC remains elusive. However, it is known that levels of nutrients and insulin, which increase in the postprandial state, induce $56 \mathrm{~K}$ activation and thereby inhibit insulin receptor signalling ${ }^{(27)}$. An excess of nutrient supply and hyperinsulinaemia associated with the obese state has been attributed to a constitutive activation of mTOR/S6K1 in adipose tissue, muscle and liver and consequently to the desensitisation of insulin signalling in mice ${ }^{(28)}$. These mechanisms may be partially triggered by the postprandial activation of $\mathrm{S} 6 \mathrm{~K}$ phosphorylation observed in MNC in the present study if a similar effect is assumed for adipose tissue, muscle and liver. Future studies are needed to elucidate this assumed possible postprandial activation of $56 \mathrm{~K}$ in other tissues and the role of postprandial activation of $\mathrm{S} 6 \mathrm{~K}$ phosphorylation in MNC under both physiological and pathophysiological conditions such as insulin resistance. Moreover, it remains elusive whether S6K activation is induced directly by insulin or other mediators that are increased postprandially, i.e. incretin hormones ${ }^{(29)}$ or metabolites ${ }^{(30)}$.

Interestingly, we did neither find a correlation of insulin levels with $\mathrm{p}$-Akt nor of $\mathrm{p}$-S6K levels with $\mathrm{p}$-Akt, despite their well-established interaction at the molecular level ${ }^{(31-33)}$. These findings may be attributed to the well-established complex interrelationship between S6K and Akt pathways, e.g. postprandial activation of $\mathrm{p}-\mathrm{S} 6 \mathrm{~K}$ may decrease Akt phosphorylation levels by negative feedback ${ }^{(34)}$ and, in turn, modulate the expression of NF- $\mathrm{kB}$ target genes. Such interactions in MNC require future analysis. Furthermore, it is well established that Akt is activated by several other stimuli besides insulin or growth factors ${ }^{(35)}$ such as T- and B-cell receptor activation ${ }^{(36,37)}$ and integrins ${ }^{(38)}$

However, we reported a negative correlation of postprandial plasma insulin levels and $\mathrm{NF}-\kappa \mathrm{B}$ activation, reflecting the well-established anti-inflammatory effects of insulin ${ }^{(39,40)}$. Strikingly, correlation analysis implies a functional relationship between postprandial inflammatory (NF- $\mathrm{B}$ ) and metabolic (Akt) signalling pathways in MNC. In fact, Akt has been shown to induce NF- $\mathrm{B}$ activation by both phosphorylation of inhibitor NF- $\mathrm{kB}$ kinase 2 in $293 \mathrm{~T}$ cells $^{(41)}$ and inhibitor $\mathrm{NF}-\kappa \mathrm{B}$ kinase 1/2-independent phosphorylation of the p65
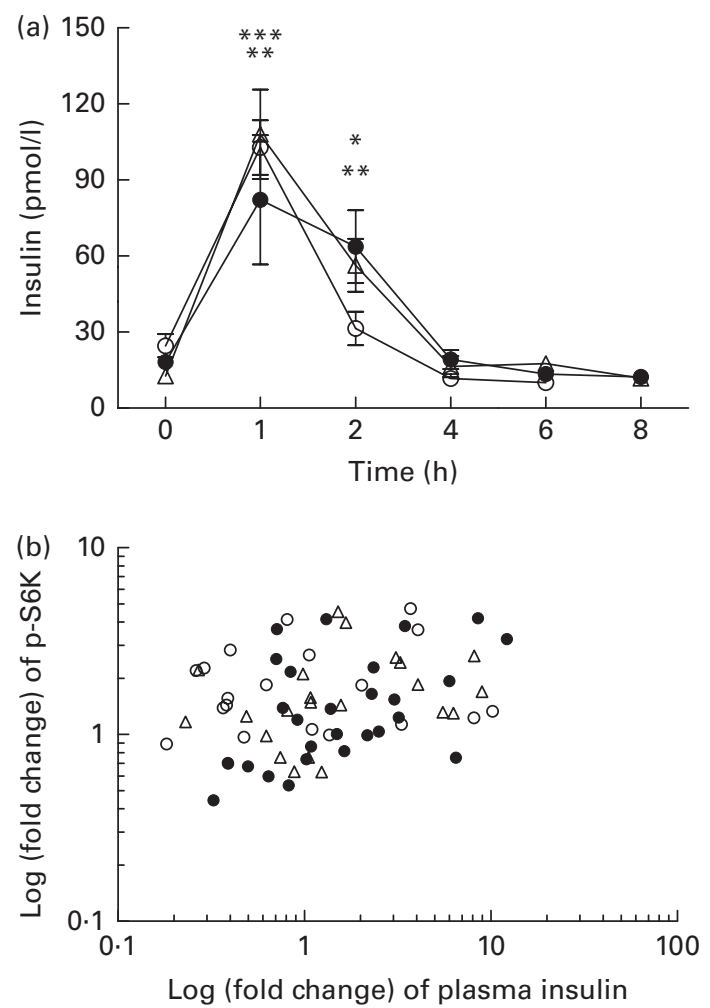

Fig. 4. Within-subject correlation of postprandial plasma insulin levels and S6 kinase (S6K) activation. (a) Plasma insulin levels (pmol/l) at baseline and following the consumption of a high-fat, high-carbohydrate (HFHC, $\triangle$ ) meal, an oral lipid-tolerance test $(\mathrm{OLTT}, \bullet)$ or a healthy breakfast $(\mathrm{HB}, \mathrm{O})$ are shown for the indicated time points. Values are means $(n 6)$, with standard errors represented by vertical bars. Mean value 1 and $2 \mathrm{~h}$ after consumption of OLTT was significantly different from that at baseline: ${ }^{*} P<0.05,{ }^{\star \star}{ }^{*} P<0.001$, respectively (one-way repeated-measures ANOVA (RMANOVA) with Bonferroni post-test). Mean value 1 and $2 \mathrm{~h}$ after consumption of HFHC meal was significantly different from that at baseline: ${ }^{\star *} P<0.001,{ }^{\star * *} P<0.0001$, respectively (one-way RMANOVA with Bonferroni post-test). Mean value $1 \mathrm{~h}$ after $\mathrm{HB}$ was significantly different from that at baseline: ${ }^{\star * \star} P<0.0001$ (oneway RMANOVA with Bonferroni post-test). There was significant time $(P<0.0001)$ and diet $(P=0.5920)$ effects (two-way RMANOVA). (b) Logtransformed fold changes of plasma insulin and $\mathrm{p}$-S6K levels for each time point and each subject are plotted against each other $(r 0.4786, P=0.0001$; within-subject correlation). 
Table 2. Within-subject correlations of intracellular signalling pathways in mononuclear cells with plasma insulin levels*

\begin{tabular}{lrr}
\hline & \multicolumn{2}{c}{ Plasma insulin } \\
\cline { 2 - 3 } Variables & \multicolumn{1}{c}{ r† } & \multicolumn{1}{c}{$P$} \\
\hline p-S6K & 0.4786 & $<0.0001$ \\
p-Akt & -0.1292 & 0.3291 \\
NF-кB binding activity & -0.3993 & 0.0016 \\
lкB- $\alpha$ & -0.0395 & 0.7682 \\
\hline
\end{tabular}

p-S6K, phosphorylated S6 kinase; p-Akt, phosphorylated Akt; І ІкB- $\alpha$, inhibitory кB- $\alpha$.

${ }^{*}$ Correlation matrix (ANCOVA): correlation coefficients within subjects of the fold change of each measured marker (oral lipid-tolerance test, high-fat, high-carbohydrate meal and healthy breakfast) at each time point and each subject were calculated.

† ANCOVA.

subunit in $\mathrm{NIH}-3 \mathrm{~T}^{(42)}$ and primary mouse embryo fibroblasts $^{(43)}$. Moreover, more recently and related to the present findings in MNC, Akt signalling has been shown to be involved in T-cell activation by interfering with NF- $\mathrm{B}$ activation $^{(36)}$. Interestingly, Akt dampens but does not impair NF- $\kappa \mathrm{B}$ binding activity in T cells. Furthermore, several studies conducted on mice have indicated a contribution of MNC to the development of systemic insulin resistance, e.g. mice with a myeloid lineage-specific deletion of insulin receptor or inhibitor NF- $\mathrm{BB}$ kinase 2 are protected against the development of diet-induced insulin resistance, and both
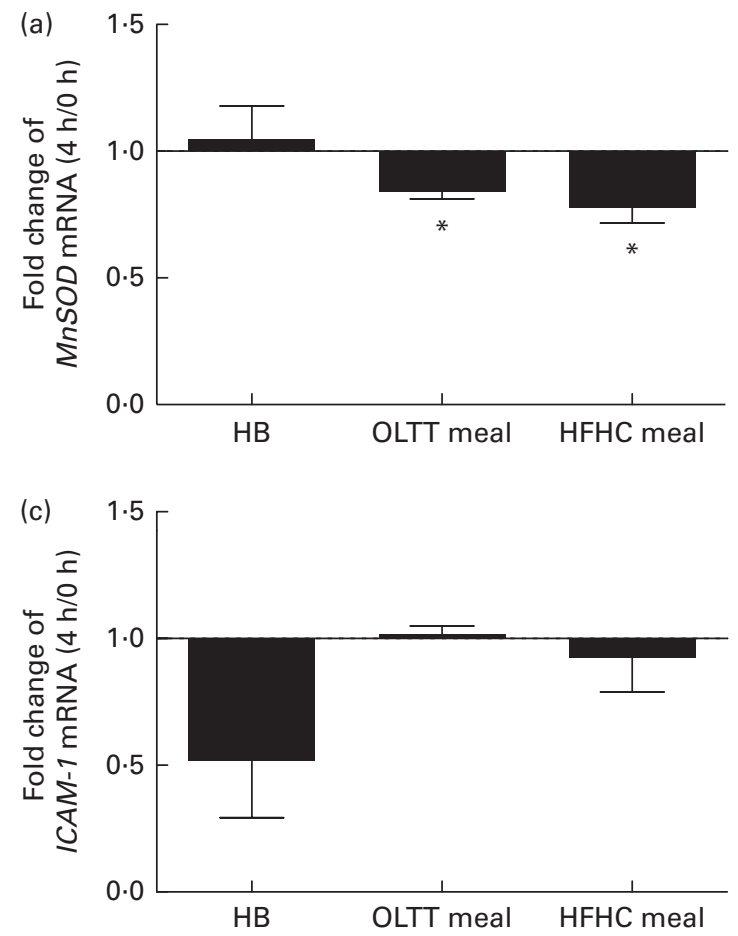

lineages exhibit a dramatic reduction in chronic and systemic low-grade inflammation associated with obesity ${ }^{(44,45)}$. Overall, these data support a combined mode of action of inflammatory activation and impaired Akt signalling in MNC; however, the precise biological function of the postprandial correlation observed between p-Akt and NF- $\mathrm{BB}$ activation in the present study remains elusive. Even if we show that there is no difference in the temporal pattern of the activation of both pathways, future studies are needed to elucidate the molecular mechanisms underlying the postprandial inference of both pathways as well as factors responsible for activation, i.e. endocrine pathways or metabolites.

The expression of the FOXO target gene MnSOD was down-regulated after ingestion of the OLTT meal and HFHC meal, but not the $\mathrm{HB}$, reflecting the known PI3K (phosphatidylinositide 3-kinase)/Akt signalling-mediated decrease in FOXO activity and FOXO target gene expression ${ }^{(46)}$. Regulation of the major mitochondrial antioxidant enzyme MnSOD suggests that postprandial Akt phosphorylation may be involved in the modulation of cellular reactive oxygen species levels. In fact, intake of HFHC meals has been shown to increase MnSOD protein levels ${ }^{(47)}$ and, compared with a meal rich in fibre and fruit, to induce oxidative stress $^{(4)}$. Furthermore, expression levels of the NF-кB target gene CCR5 were increased after ingestion of the OLTT meal compared with the $\mathrm{HB}$, implying that consumption of a meal enriched with lipids compared with a well-balanced meal may contribute to the migration of MNC into adipose tissue.
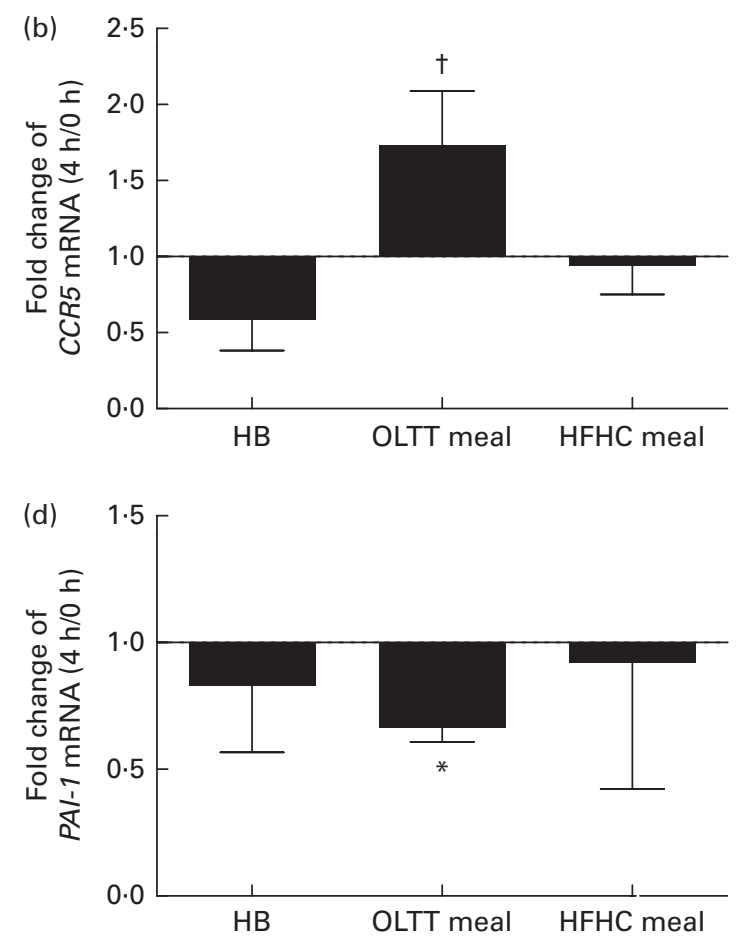

Fig. 5. Gene expression analysis of selected target genes. Mean effects of the test meals consumed on the gene expression of selected target genes of NF- $\mathrm{B}$ and Forkhead box $\mathrm{O}$ after $4 \mathrm{~h}$ compared with baseline. (a) Manganese superoxide dismutase (MnSOD), (b) CC-chemokine-receptor 5 (CCR5), (c) intercellular adhesion molecule $1(I C A M-1)$ and (d) plasminogen activator inhibitor-1 (PAl-1). Values are means, with their standard errors represented by vertical bars. * Mean value was significantly different from that at baseline $(0 \mathrm{~h})(P<0.05$; Wilcoxon matched-pairs test and Kruskal-Wallis ANOVA). $†$ Mean value was significantly different from that of the healthy breakfast $(\mathrm{HB})$ at a $4 \mathrm{~h}(P<0.05$; Wilcoxon matched-pairs test and Kruskal-Wallis ANOVA). OLTT meal, oral lipid-tolerance test meal; HFHC meal, high-fat, high-carbohydrate meal. 
In studies conducted on human subjects, an enhanced expression level of CCR5 has been found in adipose tissue $^{(48)}$, and the CCR5 ligand CCL5 has been shown to trigger the adhesion and transmigration of blood monocytes through endothelial cells of human white adipose tissue ${ }^{(49)}$. Unexpectedly, as the expression of the NF-кB target gene PAI-1 has been shown to increase in adipose tissue and macrophages in obesity and diabetes ${ }^{(50)}$, we found that gene expression levels of $P A I-1$ in MNC were reduced in response to all the three test meals studied.

The present exploratory study included a small sample size, leading to limitations regarding the direct comparison of signalling response to the HB, HFHC meal and OLTT meal. Specifically, we observed a high inter-individual variation in postprandial NF- $\kappa \mathrm{B}$ activation, I $\mathrm{KB}-\alpha$ protein degradation and both $\mathrm{S} 6 \mathrm{~K}$ and Akt phosphorylation. A post hoc power calculation revealed a required sample size of nine subjects for NF- $\kappa \mathrm{B}$ activation and Akt phosphorylation and of eleven subjects for IкB- $\alpha$ protein degradation and S6K phosphorylation to reach a statistical significant result with a power of $80 \%$ for each test meal. It should be noted that, due to the study design, we could not compare the measured activation of metabolic and inflammatory signalling pathways $8 \mathrm{~h}$ after the intake of the HFHC meal and OLTT meal with the HB in the present study. Moreover, the complexity of the test meals including differing energy content, macronutrient composition, volume and inclusion of solid and liquid meals limits the interpretation of what aspects of the test meal contribute to the observed effects.

In summary, we show herein for the first time an increase of Akt and S6K phosphorylation levels in MNC after three defined test meals. The suggested interaction of postprandial Akt and NF-кB activation may contribute to the further understanding of the interaction between postprandial immune system and metabolism.

\section{Supplementary material}

To view supplementary material for this article, please visit http://dx.doi.org/10.1017/S0007114514000208

\section{Acknowledgements}

The authors thank Manuela Hubersberger and Elisabeth Hofmair for excellent technical assistance, Cornelia Brunner for expert advice on the electrophoretic mobility shift assay of NF- $\mathrm{B}$ and Ina Rondak for statistical advice.

The present study was supported by the Else KrönerFresenius Foundation, Bad Homburg v. d. H., Germany, by a grant from the German Federal Ministry of Education and Research (BMBF, grant no. 0315494A) (project SysMBo) and by the grant Clinical Cooperation Group 'Nutrigenomics and Type 2 Diabetes' received from the Helmholtz Zentrum München, München-Neuherberg, Germany, and the Technische Universität München, Freising-Weihenstephan, Germany. The funders had no role in the study design, data collection and analysis, decision to publish, or preparation of the manuscript.
The authors' responsibilities were as follows: H. H., H. L., T. B. and K. E. designed the research; K. E., T. B. and A. B. performed the research; K. E. and H. L. analysed the data; K. E. and H. L. wrote the manuscript. All authors read and approved the final manuscript.

There are no conflicts of interest.

\section{References}

1. van Oostrom AJHHM, Rabelink TJ, Verseyden C, et al. (2004) Activation of leukocytes by postprandial lipemia in healthy volunteers. Atherosclerosis 177, 175-182.

2. Gower RM, Wu H, Foster GA, et al. (2010) CD11c/CD18 expression is upregulated on blood monocytes during hypertriglyceridemia and enhances adhesion to vascular cell adhesion molecule-1. Arterioscler Thromb Vasc Biol 31, 160-166.

3. Dandona P, Ghanim H, Chaudhuri A, et al. (2010) Macronutrient intake induces oxidative and inflammatory stress: potential relevance to atherosclerosis and insulin resistance. Exp Mol Med 42, 245.

4. Ghanim H, Abuaysheh S, Sia CL, et al. (2009) Increase in plasma endotoxin concentrations and the expression of Toll-like receptors and suppressor of cytokine signaling-3 in mononuclear cells after a high-fat, high-carbohydrate meal: implications for insulin resistance. Diabetes Care 32, 2281-2287.

5. Ghanim H, Aljada A, Daoud N, et al. (2007) Role of inflammatory mediators in the suppression of insulin receptor phosphorylation in circulating mononuclear cells of obese subjects. Diabetologia 50, 278-285.

6. Ueki K, Kondo T \& Kahn CR (2004) Suppressor of cytokine signaling 1 (SOCS-1) and SOCS-3 cause insulin resistance through inhibition of tyrosine phosphorylation of insulin receptor substrate proteins by discrete mechanisms. Mol Cell Biol 24, 5434-5446.

7. Li L, Naples M, Song H, et al. (2007) LCAT-null mice develop improved hepatic insulin sensitivity through altered regulation of transcription factors and suppressors of cytokine signalling. Am J Physiol Endocrinol Metab 293, E587-E594.

8. Rui L, Yuan M, Frantz D, et al. (2002) SOCS-1 and SOCS-3 block insulin signaling by ubiquitin-mediated degradation of IRS1 and IRS2. J Biol Chem 277, 42394-42398.

9. Kim J, Kim JE, Liu H, et al. (2008) Regulation of interleukin6-induced hepatic insulin resistance by mammalian target of rapamycin through the STAT3-SOCS3 pathway. J Biol Chem 283, 708-715.

10. Houstis N, Rosen ED \& Lander ES (2006) Reactive oxygen species have a causal role in multiple forms of insulin resistance. Nature 440, 944-948.

11. Kim JK, Kim Y, Fillmore JJ, et al. (2001) Prevention of fatinduced insulin resistance by salicylate. J Clin Invest $\mathbf{1 0 8}$, $437-446$.

12. Shoelson SE, Lee J \& Yuan M (2003) Inflammation and the $\mathrm{IKK} \beta / \mathrm{I} \kappa \mathrm{B} / \mathrm{NF}-\kappa \mathrm{B}$ axis in obesity- and diet-induced insulin resistance. Int J Obes Relat Metab Disord 27, Suppl. 3, S49-S52.

13. MacIver NJ, Jacobs SR, Wieman HL, et al. (2008) Glucose metabolism in lymphocytes is a regulated process with significant effects on immune cell function and survival. J Leukoc Biol 84, 949-957.

14. Di Paolo S, Teutonico A, Leogrande D, et al. (2006) Chronic inhibition of mammalian target of rapamycin signaling downregulates insulin receptor substrates 1 and 2 and AKT activation: a crossroad between cancer and diabetes? $\mathrm{J} \mathrm{Am}$ Soc Nephrol 17, 2236-2244. 
15. Zoncu R, Efeyan A \& Sabatini DM (2011) mTOR: from growth signal integration to cancer, diabetes and ageing. Nat Rev Mol Cell Biol 12, 21-35.

16. Thomson AW, Turnquist HR \& Raimondi G (2009) Immunoregulatory functions of mTOR inhibition. Nat Rev Immunol 9, 324-337.

17. Pierau M, Na S, Simma N, et al. (2012) Constitutive Akt1 signals attenuate B-cell receptor signaling and proliferation, but enhance B-cell migration and effector function. Eur $J$ Immunol 42, 3381-3393.

18. Lee YG, Lee J, Byeon SE, et al. (2011) Functional role of Akt in macrophage-mediated innate immunity. Front Biosci 16, 517-530.

19. Kim SY, Jeong E, Joung SM, et al. (2012) PI3K/Akt contributes to increased expression of Toll-like receptor 4 in macrophages exposed to hypoxic stress. Biochem Biophys Res Commun 419, 466-471.

20. Beugnet A, Tee AR, Taylor PM, et al. (2003) Regulation of targets of mTOR (mammalian target of rapamycin) signalling by intracellular amino acid availability. Biochem J 372, 555 .

21. Falkenberg VR, Whistler T, Murray JR, et al. (2011) Identification of phosphoglycerate kinase 1 (PGK1) as a reference gene for quantitative gene expression measurements in human blood RNA. BMC Res Notes $4,324$.

22. Bland JM \& Altman DG (1995) Calculating correlation coefficients with repeated observations: part 1 - correlation within subjects. BMJ 310, 446.

23. Faul F, Erdfelder E, Lang A, et al. (2007) G*Power 3: a flexible statistical power analysis program for the social, behavioral, and biomedical sciences. Behav Res Methods 39, 175-191.

24. Faul F, Erdfelder E, Buchner A, et al. (2009) Statistical power analyses using $G^{*}$ Power 3.1: tests for correlation and regression analyses. Behav Res Methods 41, 1149-1160.

25. Stagakis I, Bertsias G, Karvounaris S, et al. (2012) Anti-tumor necrosis factor therapy improves insulin resistance, beta cell function and insulin signaling in active rheumatoid arthritis patients with high insulin resistance. Arthritis Res Ther 14 , R141.

26. Pasini E, Flati V, Paiardi S, et al. (2010) Intracellular molecular effects of insulin resistance in patients with metabolic syndrome. Cardiovasc Diabetol 9, 46.

27. Manning B (2003) Rheb fills a GAP between TSC and TOR. Trends Biochem Sci 28, 573-576.

28. Um SH, Frigerio F, Watanabe M, et al. (2004) Absence of S6K1 protects against age- and diet-induced obesity while enhancing insulin sensitivity. Nature 431, 200-205.

29. Kwon G, Marshall CA, Pappan KL, et al. (2004) Signaling elements involved in the metabolic regulation of mTOR by nutrients, incretins, and growth factors in islets. Diabetes 53, Suppl. 3, S225-S232.

30. Krug S, Kastenmuller G, Stuckler F, et al. (2012) The dynamic range of the human metabolome revealed by challenges. FASEB J 26, 2607-2619.

31. Inoki K, Li Y, Zhu T, et al. (2002) TSC2 is phosphorylated and inhibited by Akt and suppresses mTOR signalling. Nat Cell Biol 4, 648-657.

32. Haar EV, Lee S, Bandhakavi S, et al. (2007) Insulin signalling to mTOR mediated by the Akt/PKB substrate PRAS40. Nat Cell Biol 9, 316-323.

33. Sarbassov DD (2005) Phosphorylation and regulation of Akt/ PKB by the Rictor-mTOR complex. Science 307, 1098-1101.
34. Zhang J, Gao Z, Yin J, et al. (2008) S6K directly phosphorylates IRS-1 on Ser-270 to promote insulin resistance in response to TNF- $\alpha$ signaling through IKK2. J Biol Chem 283, 35375-35382.

35. Li X, Huang Y, Jiang J, et al. (2011) Synergy in ERK activation by cytokine receptors and tyrosine kinase growth factor receptors. Cell Signal 23, 417-424.

36. Cheng J, Phong B, Wilson DC, et al. (2011) Akt fine-tunes NF- $\kappa \mathrm{B}-$ dependent gene expression during $\mathrm{T}$ cell activation. J Biol Chem 286, 36076-36085.

37. So L, Yea SS, Oak JS, et al. (2013) Selective inhibition of phosphoinositide 3-kinase $\mathrm{p} 110 \alpha$ preserves lymphocyte function. J Biol Chem 288, 5718-5731.

38. Kim GW, Kim H, Cho K, et al. (2009) The role of MMP-9 in integrin-mediated hippocampal cell death after pilocarpineinduced status epilepticus. Neurobiol Dis 36, 169-180.

39. Dandona P, Aljada A, Mohanty P, et al. (2001) Insulin inhibits intranuclear nuclear factor $\kappa \mathrm{B}$ and stimulates $\mathrm{I} \kappa \mathrm{B}$ in mononuclear cells in obese subjects: evidence for an anti-inflammatory effect? J Clin Endocrinol Metab 86, 3257-3265.

40. Ghanim H, Korzeniewski K, Sia CL, et al. (2010) Suppressive effect of insulin infusion on chemokines and chemokine receptors. Diabetes Care 33, 1103-1108.

41. Nidai Ozes O, Mayo LD, Gustin JA, et al. (1999) NF-кB activation by tumour necrosis factor requires the Akt serine-threonine kinase. Nature 401, 82-85.

42. Madrid LV (2001) Akt stimulates the transactivation potential of the RelA/p65 subunit of NF-кB through utilization of the IкB kinase and activation of the mitogen-activated protein kinase p38. J Biol Chem 276, 18934-18940.

43. Sizemore N (2001) Distinct roles of the IкB kinase alpha and beta subunits in liberating nuclear factor $\kappa \mathrm{B}(\mathrm{NF}-\kappa \mathrm{B})$ from $\mathrm{I} \kappa \mathrm{B}$ and in phosphorylating the p65 subunit of NF- $\mathrm{BB}$. J Biol Chem 277, 3863-3869.

44. Mauer J, Chaurasia B, Plum L, et al. (2010) Myeloid cellrestricted insulin receptor deficiency protects against obesity-induced inflammation and systemic insulin resistance. PLoS Genet 6, e1000938.

45. Arkan MC, Hevener AL, Greten FR, et al. (2005) IKK- $\beta$ links inflammation to obesity-induced insulin resistance. Nat Med 11, 191-198.

46. Kops GJPL, Dansen TB, Polderman PE, et al. (2002) Forkhead transcription factor FOXO3a protects quiescent cells from oxidative stress. Nature 419, 316-321.

47. Lim S, Won H, Kim Y, et al. (2011) Antioxidant enzymes induced by repeated intake of excess energy in the form of high-fat, high-carbohydrate meals are not sufficient to block oxidative stress in healthy lean individuals. Br J Nutr 106, 1544-1551.

48. Huber J, Kiefer FW, Zeyda M, et al. (2008) CC chemokine and $C C$ chemokine receptor profiles in visceral and subcutaneous adipose tissue are altered in human obesity. J Clin Endocrinol Metab 93, 3215-3221.

49. Keophiphath M, Rouault C, Divoux A, et al. (2009) CCL5 promotes macrophage recruitment and survival in human adipose tissue. Arterioscler Thromb Vasc Biol 30, 39-45.

50. Jankun J (2011) Can inactivators of plasminogen activator inhibitor alleviate the burden of obesity and diabetes? (Review). Int J Mol Med 29, 3-11. 